\title{
Test Field for the Validation of a Multifunctional Sensor for Distributed Subsurface Monitoring of Gas Storage Areas
}

\author{
Patrick P. Neumann ${ }^{1}$, Aleksander Wosniok ${ }^{1}$, Detlef Lazik' ${ }^{2}$, and Matthias Bartholmai ${ }^{1}$ \\ ${ }^{1}$ BAM Federal Institute for Materials Research and Testing, \\ Unter den Eichen 87, 12205 Berlin, Germany, \\ patrick.neumann@bam.de \\ ${ }^{2}$ Membranbasierte Gassensoren UG - MeGaSen,
}

Am Bassin 12, 14467 Potsdam, Germany

\begin{abstract}
BAM Federal Institute for Materials Research and Testing, in cooperation with the company MeGaSen UG carries out a research project to enhance and validate an innovative approach for distributed subsurface monitoring of gas storage areas. The concept combines different measurement technologies to one multifunctional sensor: membrane-based gas measurement technology for in-situ monitoring of gases in soil and fiber optical sensing of temperature and strain (as a measure for structural change). Key aspect of the research project is the first-time validation of the system in an application relevant dimension. For this purpose a $20 \times 20 \mathrm{~m}^{2}$ test field is build. A comprehensive validation of the system is carried out by systematic variation of different parameters like position-dependent gasinjection, temperature and mechanical impact.
\end{abstract}

Keywords: distributed multifunctional sensor, subsurface monitoring, gas storage areas, membranebased gas sensing, fiber optical sensing

\section{Introduction}

One of the main unsolved issues of underground storages for, e.g., carbon dioxide, hydrogen, and natural gas (consists primarily of methane) is the comprehensive surveillance of these areas with reasonable effort and costs. Conventional sensors (e.g., soil air probes or borehole probes), however, can only be used for punctual or locally limited measurements; further their application can cause structural influences (invasive application).
In cooperation with MeGaSen (a start-up from UFZ, www.megasen.com), BAM carries out a research project to enhance and validate an innovative approach for a distributed subsurface monitoring of gas storage areas. The novel sensor layout offers the characteristics for a considerable technological advance in this crucial field of operation. The sensor installation can be designed with respect to the task or problem and in-situ maintenance of the installed sensors is possible without their dismounting.

Key aspect of the research project is the firsttime validation of the technology for spatially 
resolved gas detection in an application relevant scale. For this purpose a $20 \times 20 \mathrm{~m}^{2}$ soil test field is build.

\section{Multifunctional Sensor}

The multifunctional sensor consists of a membrane-based gas sensor for in-situ monitoring of gases in soil Fehler! Verweisquelle konnte nicht gefunden werden. and a glass / polymer fiber optical sensor to measure temperature and strain [2].

The gas sensor is based on the selective permeation of gases through a membrane. The measuring method combines the gas specific diffusion rates through a membrane with Dalton's law of partial pressures. It enables the calculation of gas concentrations based on the

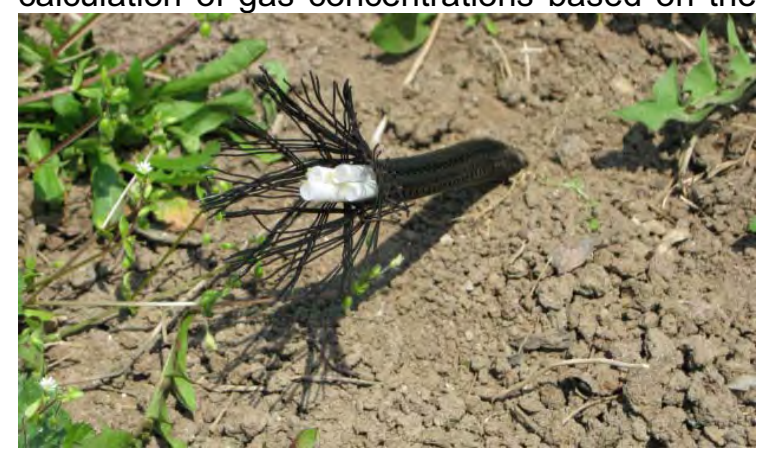

Fig. 1. Membrane-based gas sensor in soil.

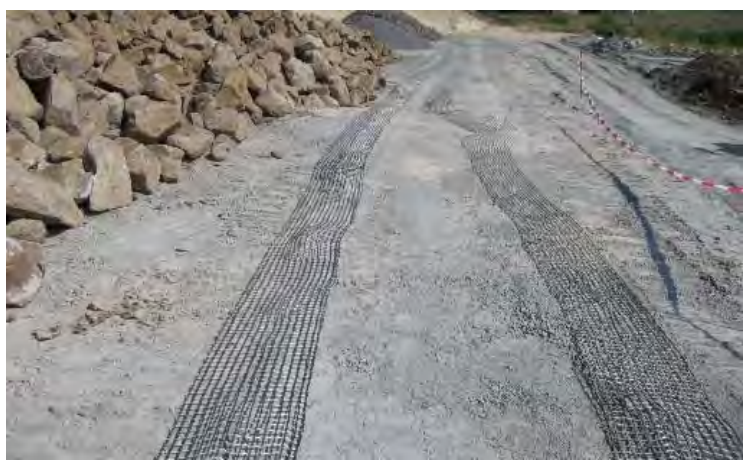

Fig. 2. Geogrid with integrated fiber optical sensors.

Fiber optical measuring systems can be implemented with length up to several kilometers with a spatial resolution in meter range.

The combination of both sensor types to one multifunctional sensor offers an innovative and promising approach for spatially resolved monitoring of large-scale areas. Using sensor data fusion will enable in-depth analysis of soil processes and early detection of relevant changes. Both technologies offer advantageous specifications [3]:

- distributed, area-wide applicable measuring system with spatially resolution of all variables ideal gas law using measurements of pressure, time, and temperature. The sensor is implemented in form of a flexible tube (Fig. 1). The synthetic material allows a variable subsurface installation, e.g., in meander or network form. So far the gas concentration measurement is implemented for carbon dioxide $\left(\mathrm{CO}_{2}\right)$ and oxygen $\left(\mathrm{O}_{2}\right)$, further gases should follow, e.g., methane $\left(\mathrm{CH}_{4}\right)$ and hydrogen sulfide $\left(\mathrm{H}_{2} \mathrm{~S}\right)$.

Glass fiber optical sensors use the effects of stimulated Raman scattering (SRS) and stimulated Brillouin scattering (SBS) for spatially resolved measuring of temperature and strain. Distributed strain measurements can also be performed with polymer optical fibers using optical time-domain reflectometry (OTDR).

- scalable and adaptable depending on monitoring object and problem

- no sensitivity against electro-magnetic fields (e.g., lightning)

- applicable in explosive surroundings (no electrical components at the measuring locations)

- high thermal and chemical robustness

- comparatively low-cost components

Geogrid materials (Fig. 2) are used as a carrier material to accomplish the linkage of both sensor types. Combined data analysis in form of data fusion should be investigated and further developed to gain synergy effects, increase the sensitivity and informational value, and address new fields of application. For instance, the combined analysis of gas concentration, temperature, and strain could enable to detect gas emissions also by crack deformation or local temperature effects, with significant higher reliability compared to sole gas measurements.

\section{Test Field}

The validation, optimization, and practical demonstration of the overall system will be carried out on the BAM 'Test Site Technical Safety' (BAM TTS) [5]. For this purpose, a test field of $20 \times 20 \mathrm{~m}^{2}$ is under construction to validate and enhance the combined system in an application relevant scale. The soil body of the test field consists of medium sand that is typical for the 'Baruther' glacial valley. The soil body will be homogenized during sensor installation. Column-like bodies of different sand grades will be implemented to observe heterogeneity depended effects on the gas movement. The sensor installation should be applied in several depth of the test field. A total of three sensor levels are planned: one sensor level should cover the complete field area, while the other 


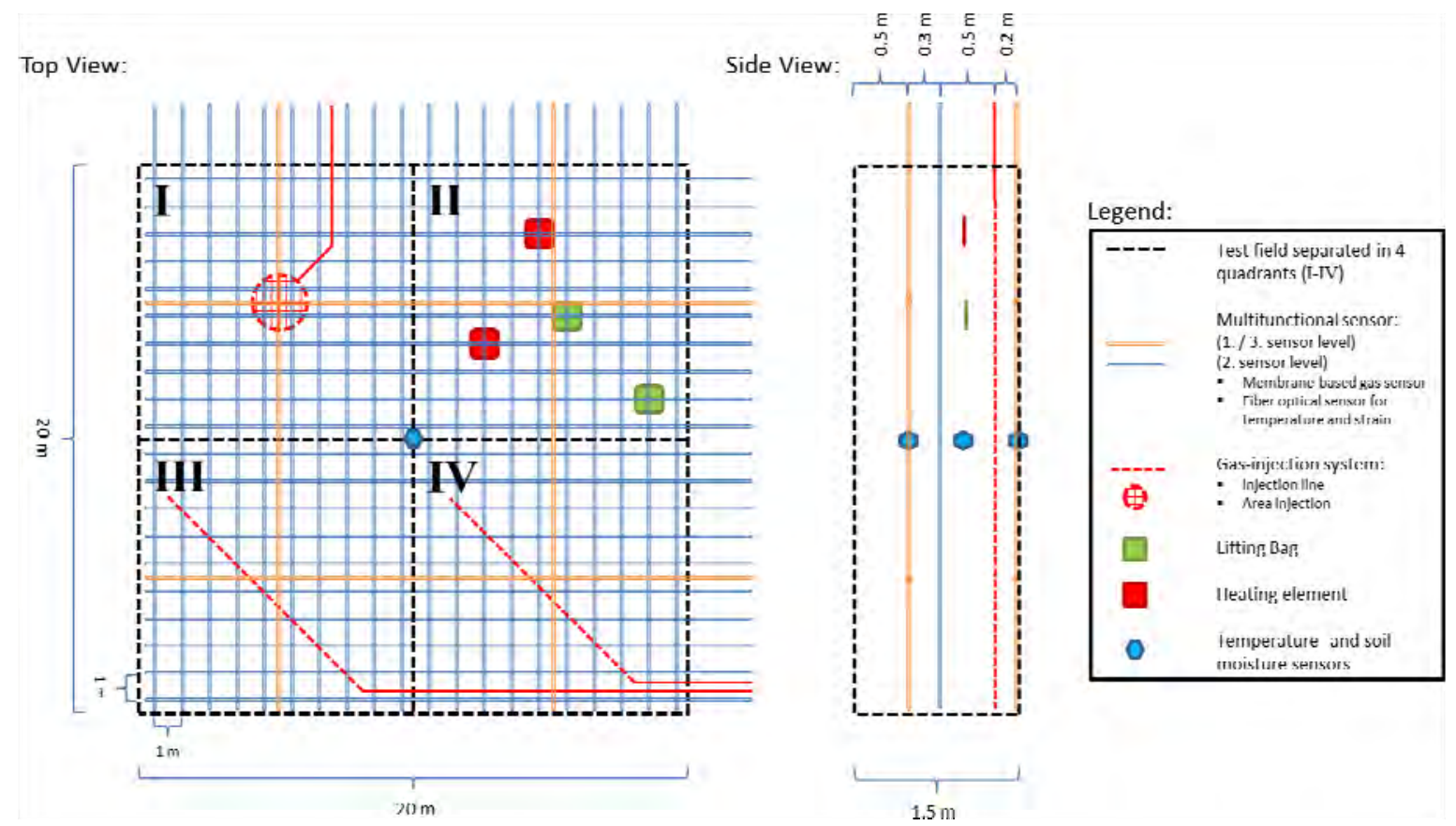

Fig. 3. Sketch of the $20 \times 20 \mathrm{~m}^{2}$ large test field consisting of three sensor levels, a gas-injection system, lifting bags to simulate structural changes, heating elements to induce a local change in temperature, and temperature and soil moisture sensors.

two should cover the area at least partially. An exact draft for the test field is shown in Fig. 3. For the simulation of gas emissions an injection system with about 100 gas injection points is implemented in a way that a quantitatively and spatially defined gas release can be applied reproducibly. A systematic variation of parameters, such as position dependent gas injection, soil temperature (through heating elements and shielding of the solar irradiation), or mechanical impact (through lifting bags) which will be repeated for various weather and ambient conditions (irrigation, wind, soil water content, etc.) allows a comprehensive investigation and validation of the multisensory system.

The equipment for system operation and data acquisition, including industrial PC is located on the surface of the test field, protected against weather and unauthorized access. The BAM TTS infrastructure for power supply and network access is available, so that a continuous operation and online data transfer are assured.

\section{Expected Results}

The following results are expected to be obtained with the test field within the project duration:

- The near real-time analysis of the local concentration distribution within the soil will be demonstrated at field scale based on an inversion of the analyzed mean concentrations by the running gas sensor grid.
- An improvement of resolution of the gas measurement can be expected based on the spatially highly resolved temperature information.

- Identification and differentiation of gas emission processes in the ground (e.g., soil respiration, seam fire, and leakage) due to different dependencies on the temperature and soil structure, or a subsequent resulting temperature and tension signature.

- Significant gain in knowledge regarding advective gas movement within soil, the emission behavior of different soil structures, and dependencies between the features of the gas source (emission rate and shape) and gas movement.

- Additional gain in knowledge regarding the temperature distribution and soil structure changes as a result of sediment movements, settlement phenomena, rearrangement of the soil moisture, etc.

- In-situ calibration as demonstrated in [4] assures the long-term operability of the sensor and enables to observe the ageing behavior of the membrane material.

\section{Fields of Application}

Two immediate fields of application of the multifunctional sensor are landfills and underground gas storage areas: 
Landfills produce greenhouse gas and thermal energy. The combination of both measurement methods should allow a potent landfill monitoring by measuring at chemical active areas and leakages.

Underground storage of $\mathrm{CO}_{2}$ as well as extraction and production of gases from geological areas can lead to mechanical changes of the deck rock (lowering / elevation) which could lead to a regional tension field that is build up. Thus gas-leading gaps can be formed which cause local ground structure changes. The simultaneous measurement of spatially resolved gas concentrations and strain allows the development of an efficient early warning system.

\section{Summary and Outlook}

An innovative approach was introduced for a multifunctional sensor to investigate subsurface processes. The combination of the measurands gas concentration, temperature, and strain qualifies the sensor particularly for safety application in Carbon Capture and Storage (CCS) areas and landfills. Two measuring principles, the permeation-selective membrane and the stimulated Brillouin scattering (SBS) in fiber optics complement each other to a highly sophisticated sensing concept. At BAM a project is carried out in which scope a test field in application-relevant dimensions is build up. Gas emission processes can be simulated as well as temperature and mechanical impact to validate and enhance the multifunctional sensor.

Moreover, algorithms are in development for the near real-time safety assessment based on data fusion and combined analysis of the three measurands. The objective is to qualify the overall system for achieving a gain in knowledge of phenomena within the subsurface, particularly with respect to gas release processes. Future tasks are the long-term validation of the sensing characteristics such as robustness, sensitivity, reliability, and the enhancement to measure other relevant gases such as $\mathrm{CH}_{4}$ or $\mathrm{H}_{2} \mathrm{~S}$.

Furthermore, the test field will enable the possibility to perform ground truth validated experiments in natural environments for gas measuring methods at ground level, e.g., with gassensitive mobile robots, such as the one presented in [6].

\section{Acknowledgements}

The authors thank the participating colleagues from BAM and MeGaSen, by name W. Daum, M. Kammermeier, H. Kohlhoff, K.-D. Werner, K. Krebber, and S. Ebert. The authors also express their gratitude to the German Federal Ministry of Economics and Technology for funding the research (MNPQ Program; file number 17/11).

\section{References}

[1] Lazik, D.; Ebert, S.; Leuthold, M.; Hagenau, J.; Geistlinger, H.; Membrane Based Measurement Technology for in situ Monitoring of Gases in Soil. Sensors 2009, 9, 756-767.

[2] Liehr, S.; Lenke, P.; Wendt, M.; Krebber, K.; et al.; Polymer optical fiber sensors for distributed strain measurement and application in structural health monitoring. IEEE Sensors Journal 9, 2009, $11,1330-1338$

[3] Bartholmai, M.; Neumann, P. P.; Lazik, D.; Multifunctional Sensor for Monitoring of $\mathrm{CO}_{2}$ Underground Storage by Comprehensive and Spatially Resolved Measuring of Gas Concentrations, Temperature and Structural Changes, in Energy Procedia, 2012, to appear.

[4] Lazik, D.; Ebert, S.; Improved membrane-based sensoral set-up for a reliable gas monitoring in the subsurface. Sensors, submitted 2012.

[5] http://www.tts.bam.de

[6] Neumann, P. P.; Asadi, S.; Schiller, J. H.; Lilienthal, A. J.; Bartholmai, M.; Micro-Drone for Wind Vector Estimation and Gas Distribution Mapping. IEEE Robotics and Automation Magazine, vol. 19, no. 4, pp. 50-61, 2012. 